\title{
Insect chemical communication systems
}

\author{
J. H. Tumlinson \\ Insect Attractants, Behavior \& Basic Biology Research Laboratory, \\ Agricultural Research Service., U. S. Department of Agriculture, \\ Gainesville, Florida 32604 USA
}

\begin{abstract}
The structures of insect pheromones vary with different orders, families, genera, and species. In general, species in the same family and order have pheromones of similar structure. In the moths (Lepidoptera) the pheromone structures are fatty acid derived molecules and the biosynthetic pathways have been elucidated for many of the moth pheromones. However, the existing theory on pheromone biosynthesis in Lepidoptera cannot account for some of the more unusual structures. In contrast, the tephritid fruit fly (Diptera) pheromone structures are quite different from those of the moths and even within a single species pheromone molecules that appear to have arisen by different biosynthetic routes may be employed. Insect chemical communication systems are complex and provide a number of new compounds for structural and biosynthetic investigations.
\end{abstract}

\section{INTRODUCTION}

Many contrasts are evident between the pheromonal communication systems of moths and those of fruit flies in the family Tephritidae. The pheromone biosynthetic system of the moths is conservative in that most female moths emit blends of similar compounds to lure conspecific males. Of ten specificity in these blends is achieved by very slight variations in olefinic bond positions or geometry, by changes in the ratios of components, or by varying the functional group of one or more components. The investigations of moth pheromone biosynthesis conducted thus far indicate that in most cases hexadecanoates and octadecanoates are synthesized de novo in the pheromone glands from acetyl-CoA and malonyl-CoA, and subsequently olefinic bonds are added by desaturases, chains are shortened by beta-oxidation, and the functional groups are converted to alcohols, acetates, or aldehydes to produce the various blends characteristic of each species (ref. 1,2). However, there are still many gaps in our knowledge of pheromone biosynthesis in moths despite the seeming uniformity throughout the group.

Although only a few pheromones have been identified for the tephritid fruit flies it is already evident that there is much more diversity in these systems than in those of the moths. In most of the tephritid fruit fly systems that have been studied, the pheromone is produced by the males. The chemical structures of the pheromones vary greatly among the species, and also the volatile blend emitted by males of a species may be composed of very different types of compounds. Although little is known about the mechanisms of pheromone biosynthesis in these fruit flies, it is evident that a variety of pathways must be employed.

It is interesting that these two groups of insects have developed quite different chemical systems to accomplish the same goals. These are only two examples of the many different chemical communication systems employed by insects. Investigation of the contrasting systems should provide greater insight into the biochemistry of these vital life processes for insects. In this paper I report recent discoveries in these two groups that illustrate the diversity in the chemistry and biochemistry of insect chemical communication systems.

\section{Heliothis PHEROMONE BIOSYNTHESIS}

A good example of moth pheromone systems is provided by species in the genus Heliothis. The majority of components in the pheromone blends of species in this genus are aldehydes. The blend emitted by female $\underline{H}$. virescens is shown in Table 1 (ref. 3 ). It is evident that $\underline{H}$. virescens pheromone gland extracts contain alcohols that are not emitted by the females but that are structural congeners of the emitted aldehydes. One of the key questions in investigations of pheromone biosynthesis has been how some insects produce aldehydic pheromones since of ten these aldehydes are present in very small quantities or not at all in extracts of glands. Usually aldehydic pheromones can be obtained in significant quantities only by extracting the glands during the time when the insects are signaling. 
TABLE 1. Composition of pentane extracts of excised pheromone glands and of volatiles collected from calling Heliothis virescens females.

\begin{tabular}{lcc}
\hline Compound & $\begin{array}{l}\text { Percent } \\
\text { gland extract }\end{array}$ & $\begin{array}{l}\text { Percent }^{1} \text { in } \\
\text { female volatiles }\end{array}$ \\
\hline Tetradecanal & $2.5( \pm 0.13)$ & $13.0( \pm 0.65)$ \\
(Z)-9-Tetradecenal & $3.3( \pm 0.38)$ & $18.1 \pm 1.09)$ \\
Hexadecanal & $8.6( \pm 0.71)$ & $7.3( \pm 0.59)$ \\
$(\underline{Z})-7-$ Hexadecenal & $0.6( \pm 0.31)$ & $0.6( \pm 0.1)$ \\
$(\underline{Z})-9$-Hexadecenal & $0.8( \pm 0.15)$ & $1.0( \pm 0.1)$ \\
(Z)-11-Hexadecenal & $79.5( \pm 0.84)$ & $60.0( \pm 1.32)$ \\
Tetradecanol & $0.3( \pm 0.12)$ & \\
(Z)-9-Tetradecenol & $0.3( \pm 0.22)$ & \\
Hexadecanol & $0.4( \pm 0.25)$ & \\
(Z)-11-Hexadecenol & $4.8( \pm 0.71)$ & \\
\hline
\end{tabular}

${ }^{1}$ Data in parentheses $\pm S E$ of the mean.

Since the alcohols corresponding to the $\underline{H}$. virescens aldehydic pheromone components were found in pheromone gland extracts it seemed likely that these alcohols were biosynthetic precursors of the aldehydes. This was confirmed by application of dimethyl sulfoxide solutions of a variety of primary alcohols to the gland surface. In every case, the alcohol was converted to the aldehyde (ref. 4). The ratio of aldehydes produced was the same as the ratio of alcohols applied to the gland. Subsequent studies showed that the alcohols were converted to aldehydes by a nonspecific primary alcohol oxidase (ref. 4) and that this oxidase resides in the cuticle overlying the pheromone gland (ref. 5). Thus, the aldehydes of the pheromone blend are produced just prior to their release into the atmosphere when the alcohols are excreted through the gland cuticle. The same mechanism was found to occur in $\underline{H}$. $\underline{\text { zea and }} \underline{\mathrm{H}}$. subflexa. However, $\underline{\mathrm{H}}$. subflexa also produces and emits acetates as part of the pheromone blend (ref. 6 ). Acetates applied to the gland of $\underline{H}$. subflexa were converted to alcohols which were subsequently oxidized to aldehydes. Application of alcohols to $\underline{H}$. subflexa glands resulted in the production of aldehydes but not acetates. Thus acetates apparently precede alcohols and aldehydes in the pheromone biosynthetic pathway in this insect. Interestingly, acetates applied to $\underline{H}$. virescens glands were also converted to alcohols and then aldehydes. However acetates could not be found in $\underline{\mathrm{H}}$. virescens gland extracts (ref, 7,8). This anomaly is not yet clearly understood. We are continuing to investigate the biosynthesis of Heliothis pheromones, and have made progress toward isolating and purifying the primary alcohol oxidase.

\section{Manduca sexta PHEROMONE}

Although the $\mathrm{M}$. sexta pheromone blend is also composed of aldehydes, some of which are identical to components of the $\underline{H}$. virescens pheromone, there are distinct differences between the two blends. Capillary GC and GC-MS analyses of hexane washes of $\underline{M}$. sexta female pheromone glands revealed the presence of a series of 16- and 18-carbon aldehydes including hexadecanal, $(Z)-9$-hexadecenal, $(Z)$ - and $(E)$-11-hexadecenal, $(\underline{E}, \underline{E})$ - and $(\underline{E}, \underline{Z})-10,12$-hexadecadienal $(\underline{1})$, octadecanal, $(\underline{Z})$-1I-octadecenal, $(\underline{Z})$-13-octadecenal, and $(\underline{Z}, \underline{Z})-11,13$ octadecadienal (ref. 9 ). Previously (E, $\underline{Z})-10,12$-hexadecadienal (bombykal) (1 $)$ was reported to be a sex pheromone of $\underline{\underline{M}}$. sexta (ref. 10), but it is only slightly active in wind tunnel bioassays, and does not elicit the complete repertoire of responses from males. Similarly, a synthetic blend of the above listed compounds formulated in the same ratio that they were found in hexane gland washes was only slightly attractive to male $\underline{M}$. sexta in a wind tunnel. Two additional compounds, $(\underline{E}, \underline{E}, \underline{Z})-$ and $(\underline{E}, \underline{E}, \underline{E})-10,12,14-$ hexadecatrienal, (2) and (3), respectively, were isolated from the gland washes by reverse phase HPLC and identified by chemical ionization mass spectroscopy and ozonolysis (ref. 9). The identity of these compounds was confirmed by synthesis. The trienes are very unstable and significant losses are incurred when purification of the natural compounds is attempted by micropreparative GC. Both (1) and (2) are required for attraction of $\underline{\mathrm{M}}$. sexta males. Other components appear to have more subtle roles in the pheromonal communication system.<smiles>[CH2+]CC/C=C\C=C\CCCCCCCCCC=O</smiles>

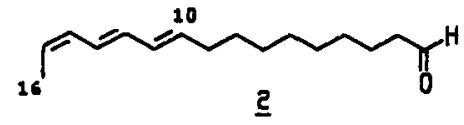

\section{M.}

Since the $\underline{M}$. sexta pheromone gland produces both $(\underline{Z})-11$-hexadecenal and bombykal it is tempting to speculate that it produces the conjugated dienes by a mechanism similar to that reported for Bombyx mori (ref. 11). However, the conjugated trienes may be more difficult to account for. Similarly, biosynthesis of the aldehydes by a mechanism similar to that in Heliothis is possible since preliminary experiments have shown that primary alcohols applied to the gland are converted into aldehydes. Investigation of this biosynthetic pathway is in progress. 


\section{FRUIT FLY PHEROMONES}

The diversity in tephritid fruit fly sex pheromones is illustrated by comparing the pheromones produced by males of three species. Chemically, the simplest pheromone is that emitted by papaya fruit fly (PFF) males. It consists of a single compound, 2-methyl-6-vinylpyrazine (4) (ref, 12) and is attractive to females in a laboratory wind tunnel and in the field. Synthetic (4) is very effective as a lure for female PFF when it is employed in the field in spherical traps of about the same size as a mature papaya fruit (ref. 13).

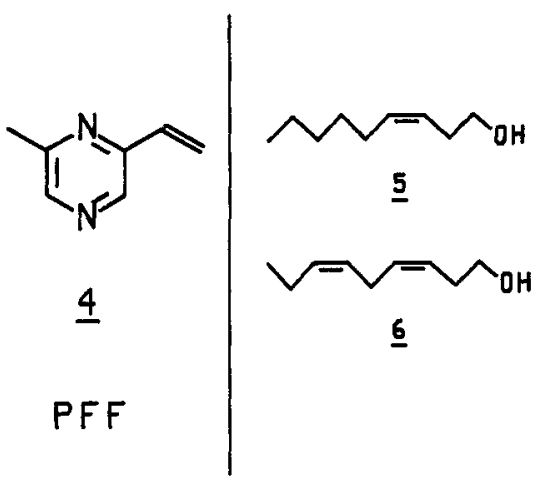

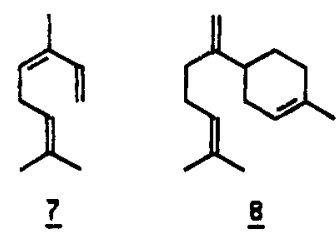<smiles>CC1=CCCOC(=O)CCC(C)=CCC1</smiles>

$\underline{9}$

$\underline{10}$

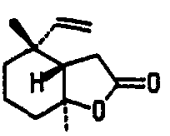

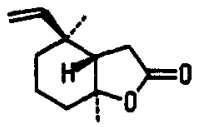

11

CFF

The blend of compounds emitted by Caribbean fruit fly (CFF) males is the most complex found in fruit flies

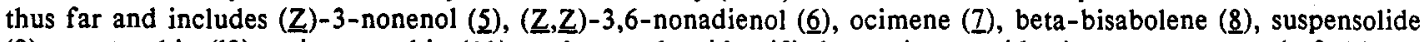
$(\underline{9})$, anastrephin (10), epianastrephin (11), and several unidentified sesquiterpenoid minor components (ref. 1416). Collection and analysis of volatiles from CFF males during different periods of the photophase indicated that the ratio of $(2)$ in the blend decreases and that of $(10)$ and $(11)$ increases as the day progresses (ref. 16). This suggests that suspensolide may be a biosynthetic precursor of the anastrephins. Suspensolide (2) is partially converted to a mixture of $(\underline{10})$ and $(11)$ by GC on packed columns. It is difficult to develop a single hypothetical biosynthetic scheme that will account for all the components of the volatiles from CFF males. It appears that CFF males are producing three structurally distinct groups of compounds. It is possible that not all of the components of CFF male volatiles are pheromones. The role of all the components in pheromonally mediated behavior in this species awaits elucidation.

Previously several compounds were identified in volatiles from male Mediterranean fruit flies (MFF) (ref. 17). We recently confirmed the presence of the three previously reported major components, geranyl acetate, (12), $(\underline{E}, E)$-alpha-farnesene, $(\underline{13})$, and ethyl $(\underline{E})-3$-octenoate, $(\underline{14})$ by capillary GC and GC-MS analysis of volatiles collected from both wild and laboratory reared MFF males (ref. 18). The ratio of these three components emitted by wild MFF males differed from that emitted by the lab-reared males. We also detected several other compounds present in the volatiles in amounts of less than $1 \%$. Our methods would not have detected the 3,4-dihydro-2H-pyrrole reported in the previous study (ref. 17). In the field in Guatemala (ref. 18), spherical traps baited with a blend of $(12),(13)$ and (14) captured significantly more MFF females than did empty traps or traps baited with trimedlure, a mixture of isomers of tert-butyl 4(and 5)-chloro-2methylcyclohexane-1-carboxylate, which is the standard lure now used in traps for MFF males (ref. 17).<smiles>CC(=O)OCC=C(C)CCC=C(C)C</smiles>

$\underline{12}$<smiles>C=CC(C)=CCC=C(C)CCC=C(C)C</smiles>

13<smiles>CCCC/C=C/CC(=O)OCC</smiles>

14

\section{MFF}

We know very little about the pheromone systems or biosynthetic mechanisms for pheromone production of tephritid fruit flies. The worldwide importance of these pests gives impetus to the need to investigate their chemical communication systems in more detail. Additionally, the fact that the pheromones attract the female flies should make them valuable as lures for traps around ports of entry in countries that are free of flies. Capture of introduced females before they lay their eggs, would be much more effective than capturing their progeny. For the same reason, lures for females should be much more effective than male attractants in programs aimed at population reduction by mass trapping. Investigation of pheromone biosynthesis enables us to more completely and accurately define the pheromone communication system of an insect. Hopefully, a thorough knowledge of the pheromone biosynthetic mechanisms may allow us to devise new, more effective methods for pest control in the future. 


\section{REFERENCES}

1. D. Morse and M.Meighen, in G. D. Prestwich, and G. J. Blomquist (eds.), Pheromone Biochemistry, pp. 121-158, Academic Press, New York (1987).

2. L. B. Bjostad, W. A. Wolf, and W. L. Roelofs, in G. J. Blomquist (eds.), Pheromone Biochemistry, pp. 77-120, Academic Press, New York (1987).

3. P. E. A. Teal, J. H. Tumlinson, and R. R. Heath, J. Chem. Ecol. 12, 107-126 (1986),

4. P. E. A. Teal, and J. H. Tumlinson, J. Chem. Ecol. 12, 343-366 (1986).

5. P. E. A. Teal, and J. H. Tumlinson, J. Chem. Ecol. in press (1988).

6. P. E. A. Teal, R. R. Heath, J. H. Tumlinson, and J. R. McLaughlin, J. Chem. Ecol. I, 1011-1022 (1981).

7. P. E. A. Teal, and J. H. Tumlinson, in J. Law (ed.), Molecular Entomology, pp. 79-94, Alan R. Liss, New York (1987).

8. P. E. A. Teal, and J. H. Tumlinson, Arch. Insect Biochem. Physiol. 4, 261-269 (1987).

9. J. H. Tumlinson, M. M. Brennan, R. E. Doolittle, E. R. Mitchell, B. E. Mazomenos, and D. M. Jackson, J. Chem. Ecol. submitted.

10. A. N. Starratt, K. H. Dahm, N. Allen, J. G. Hildebrand, T. L. Payne, and H. Roller, Z. Naturforsch. 34c, $9-12$ (1979).

11. L. B. Bjostad, and W. L. Roelofs, Insect Biochem. 14, 275-278 (1984).

12. T. Chuman, P. J. Landolt, R. R. Heath, and J. H. Tumlinson, J. Chem, Ecol. 13, 1979-1992 (1987).

13. P. J. Landolt, R. R. Heath, H. R. Agee, J. H. Tumlinson, and C. O. Calkins, J. Econ. Entomol. 8 , 917-941 (1988).

14. J. L. Nation, in J. Miyamoto and P.C. Kearney (eds.), IUPAC Pesticide Chemistry. Human Welfare and the Environment, 2, 109-110, Pergamon Press, New York (1983).

15. M. A. Battiste, L. Strekouski, D. P. Vanderbilt, M. Visnick, R. W. King, and J. Nation, Tetrahedron Lett. 24, 2611-2614 (1983).

16. T. Chuman, J. Sivinski, J. H. Tumlinson, R. R. Heath, and C. O. Calkins, J. Chem. Ecol. in press.

17. R. Baker, R. H. Herbert, and G. G. Grant, J. Chem. Soc. Chem. Commun. 824-825 (1985).

18. R. R. Heath, P. J. Landolt, J. H. Tumlinson, R. E. Doolittle, D. L. Chambers, J. Sivinski, and C. O. Calkins, $\underline{J}$. Chem. Ecol., in preparation.

19. M. Beroza, N. Green, S. I. Gertler, L. F. Steiner, and D. H. Miyashita, J. Agric. Food Chem. 2, 361365 (1961). 